\title{
EFECTO DEL IMPACTO ANTRÓPICO SOBRE LA DISTRIBUCIÓN DE STRAMONITA HAEMASTOMA (LINNAEUS 1766) (GASTROPODA: MURICIDAE) EN LA COSTA NORTE DE TENERIFE
}

\author{
Elsa Cuende, Sara González-Delgado* \\ María L. García, Claudia Pich \\ \& María Rodríguez
}

\section{RESUMEN}

Las zonas costeras han sido históricamente un lugar de asentamiento humano y de aprovechamiento de recursos por su elevada productividad. En las islas Canarias concretamente, el marisqueo de la "púrpura" o "cańadilla" (Stramonita haemastoma) ha sido uno de los principales motivos de explotación del intermareal isleño. El objetivo del presente estudio fue analizar el efecto del impacto antrópico sobre la distribución, abundancia y tamaño de este molusco. Para ello, se muestrearon dos zonas del norte de Tenerife, la Punta del Hidalgo y Finca El Apio, lugares muy homogéneos en sus características físicas, pero con una diferencia importante, su cercanía al núcleo urbano. Los resultados muestran diferencias en la abundancia y la talla de Stramonita haemastoma entre las dos localidades, encontrando mayor número de individuos, así como individuos de mayor tamaño, en la localidad menos impactada por el ser humano. Del mismo modo, se muestra un cambio en la distribución natural de esta especie. Este estudio podría ser una herramienta para la gestión adecuada y sostenible de este recurso, a través del conocimiento de su estado actual.

Palabras Clave: impacto humano, abundancia, talla, Stramonita haemastoma.

\section{ANTHROPIC IMPACT EFFECT ON THE DISTRIBUTION OF STRAMONITA HAEMASTOMA (LINNAEUS 1766) (GASTROPODA: MURICIDAE) IN THE NORTH COAST OF TENERIFE}

\section{Abstract}

Historically coastal areas have been a human settlement place and exploitation area for its resources and its high productivity. In the Canary Islands, one of the main reasons to exploit the islands intertidal zone has been the shellfishing of "purpura o cañadilla" (Stramonita haemastoma). The main objetive of this study was to analyze the human impact's effect on the distribution, abundance and size of this mollusk. To do so, we sampled two locations at the North of Tenerife, with similar characteristics, la Punta del Hidalgo and Finca El Apio. The results show differences on the abundance and size of Stramonita haemastoma among localities, being more abundant and larger at the less human-impacted locality. In addition, there are changes in the natural distribution of this species. This study could be useful tool for sustainable management actions of this resource throughout the knowledge of its actual status.

KEYWORDS: human impact, abundance, size, Stramonita haemastoma. 


\section{INTRODUCCIÓN}

La zona intermareal costera constituye un hábitat de alta heterogeneidad topográfica que alberga una gran riqueza y diversidad de organismos, abarcando desde el límite inferior de la bajamar hasta el límite superior de la pleamar. Su patrón de zonación está bien definido siguiendo los esquemas clásicos (Stephenson y Stephenson 1949), caracterizado por la presencia de tres bandas: supralitoral, mesolitoral e infralitoral. El supralitoral está caracterizado por la presencia del molusco Littorina striata, el mesolitoral por la dominancia del cirrípedo Chthamalus stellatus y el infralitoral por céspedes de algas rojas y pardas y una densa banda de Cystoseira abies-marina que marca el final del intermareal (Elejabeitia y Afonso-Carrillo 1994). Esta zona, continuamente bañada por las mareas, constituye un territorio único en términos ecológicos, económicos y sociales. Por ello, a lo largo de la historia, el ser humano se ha establecido en torno a estas zonas, aprovechando el espacio y haciendo uso de sus recursos.

Las diferentes especies de moluscos han constituido uno de los recursos existentes de mayor interés en el litoral en términos alimenticios, estéticos y comerciales. En este contexto una importante fuente de conocimiento histórico son los yacimientos costeros, donde los restos de organismos encontrados informan que ya en tiempos pasados se hacía uso de estos recursos (Hernández 2006). De hecho, uno de los recursos costeros utilizados tanto por los fenicios como por los romanos después fue la especie Stramonita haemastoma (Linnaeus 1766), púrpura o cañadilla, un molusco gasterópodo apreciado por su pigmento natural púrpura que adornaba las más valiosas túnicas (Tesserras 2000). En las islas Canarias y más concretamente en Tenerife, su aprovechamiento por los aborígenes queda patente en el registro arqueológico desde mucho antes de la época cristiana, hacia el siglo v a.C. (Hernández 2006).

Hoy en día el marisqueo sigue siendo practicado en todo el Archipiélago Canario, de forma recreativa para consumo propio y profesionalmente mediante su comercialización. El molusco Stramonita haemastoma es objeto de dicho marisqueo, aunque su recolección está sometida a una veda desde el año 2011 para favorecer su recuperación (BOC . $^{\circ}$ 93, Orden de 18 de mayo de 2011). En un estudio previo a esta veda, llevado a cabo en las islas Canarias en 2009 (Ramírez et al. 2009), se observó una mayor abundancia a lo largo del mesolitoral rocoso, en comparación con el infralitoral, donde el estrés por desecación y exposición al oleaje es menor y existe una mayor abundancia de alimento. Por el contrario, en las islas donde la densidad de población es mucho más alta, existe una mayor abundancia en la zona inferior del intermareal.

* Máster de Biología Marina: Biodiversidad y Conservación. Universidad de La Laguna, San Cristóbal de La Laguna, Tenerife, islas Canarias, España. Autor para la correspondencia: $\underline{\text { sa- }}$ glezdel@gmail.com. 
En el presente estudio se pretende analizar la distribución espacial y el tamaño del molusco Stramonita haemastoma en función de la cercanía de los individuos a la fuente de impacto antrópico definido por el núcleo urbano. La investigación se lleva a cabo en dos zonas del norte de la isla de Tenerife; la Punta del Hidalgo y Finca El Apio. Dichas localidades se asemejan en que su rasa intermareal está constituida mayormente por roca y la orientación de la misma es noroeste de modo que se pueden obviar diferencias en la distribución debidas a factores físicos y oceanográficos (Ramírez et al. 2008). Sin embargo, una diferencia relevante entre las localidades es la dificultad de acceso al público que presentan, siendo este el elemento clave en el diseño de nuestro estudio.

\section{MATERIALES Y MÉTODOS}

\subsection{Muestreo y ReCOlección De DATOS}

La especie objeto de este estudio es Stramonita haemastoma, un molusco gasterópodo presente en las islas Canarias que habita en el meso- e infralitoral de las costas rocosas (Ramírez et al. 2009). Los lugares muestreados para la recolección de este animal se ubicaron en dos localidades de la costa norte de la isla de Tenerife

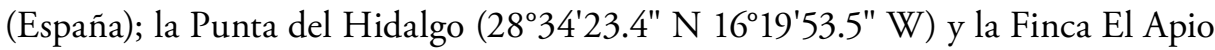

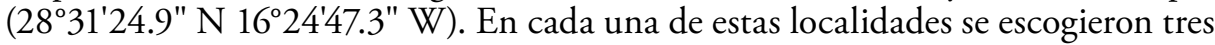
zonas de muestreo en función de su distancia a una fuente de impacto antrópico; definido por el núcleo urbano en la Punta del Hidalgo y la carretera de acceso al intermareal en la Finca El Apio. Este factor fue denominado «distancia al impacto» (DIS), siendo las zonas más cercanas «DIS 1», «DIS 2» las zonas intermedias y «DIS 3» las más alejadas (figura 1). En cada una de estas zonas se tomaron muestras en los dos niveles de marea, meso- e infralitoral, realizando tres réplicas de transectos de $10 \mathrm{~m}$ de largo y $2 \mathrm{~m}$ de ancho. Se recolectaron los individuos encontrados tanto sobre la superficie rocosa como entre grietas y oquedades del área que cubría cada transecto y se tomaron medidas de la altura de la concha (figura 2) mediante calibradores Vernier de 0,05 $\mathrm{mm}$ de precisión.

\subsection{ANÁLISIS DE LOS DATOS}

Los datos de abundancia de Stramonita haemastoma obtenidos se analizaron con el paquete estadístico PRIMER 6 Versión 6.1.11 \& PERMANOVA + Versión 1.0.1. Se realizaron análisis de la varianza por permutaciones (Anderson 2001) en función de la localidad, la cercanía al impacto humano, el nivel de marea y la interacción entre estos dos últimos. Por lo tanto, los modelos incorporaron los siguientes factores: (1) 'Localidad' (factor fijo ortogonal con dos niveles correspondientes a la Punta del Hidalgo y la Finca El Apio), (2) 'Distancia al impacto' (factor fijo ortogonal con tres niveles en función de la distancia al impacto) y (3) 'Nivel de marea' (factor fijo ortogonal con dos niveles correspondientes al infralitoral y mesolitoral). 
La Punta del Hidalgo.

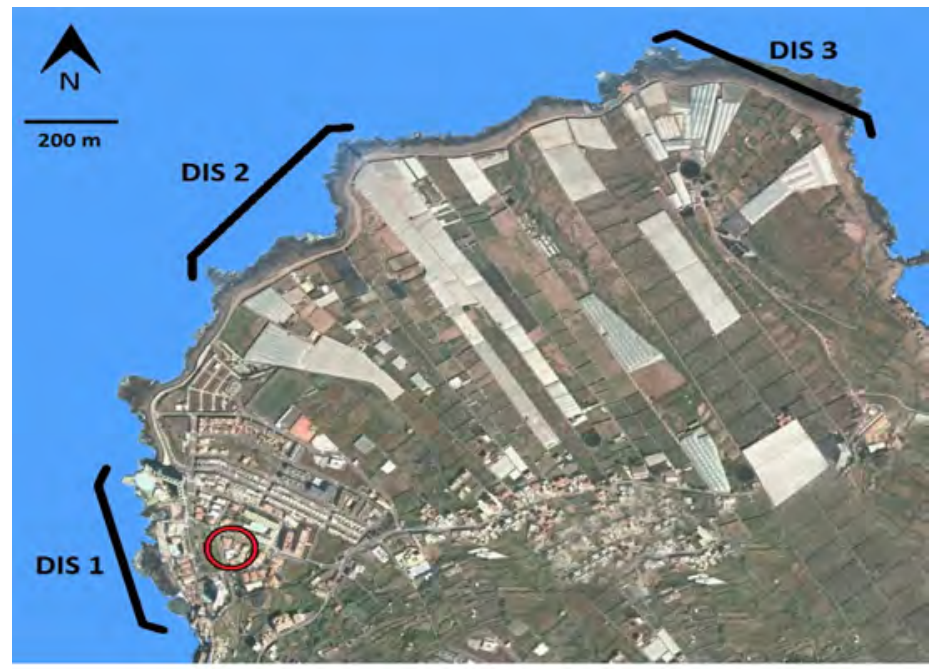

La Finca El Apio.

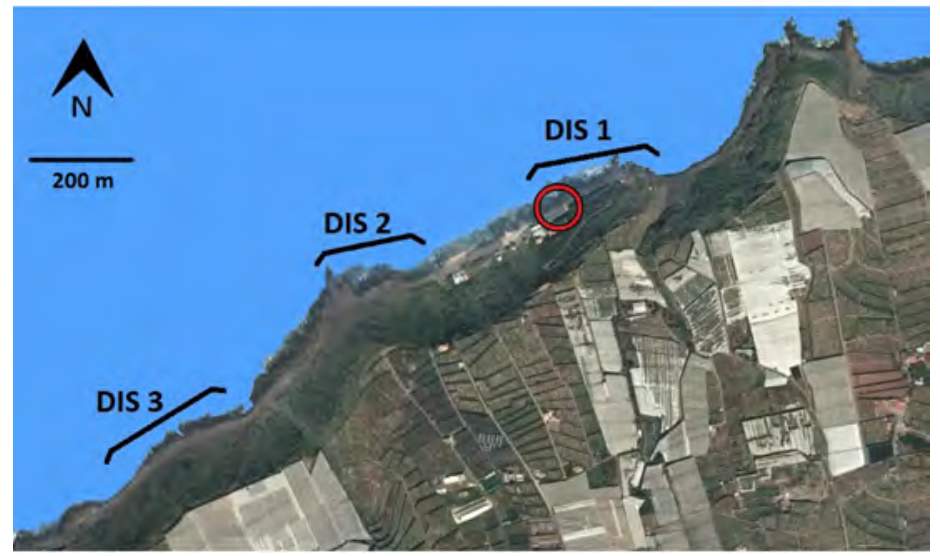

Figura 1. Zonas de muestreo en función de su distancia al acceso humano (representado por un círculo) «DIS 1», «DIS 2»y «DIS 3» en las dos localidades.

Se utilizaron las distancias euclídeas para el análisis de la variable 'Abundancia de Stramonita haemastoma'. El nivel de significación elegido fue de 0,05 y se emplearon 4999 permutaciones. Aquellos resultados significativos se representaron gráficamente usando Excel (Microsoft Office).

Por otro lado, se representaron las frecuencias de talla de los individuos de modo similar a las clases de tallas establecidos por Ramírez et al. (2009) para las dos localidades. Además, se analizaron estos datos mediante la prueba $\chi^{2}$ de independencia, con un nivel de significación de 0,01 con el software Excel (Microsoft Office). 


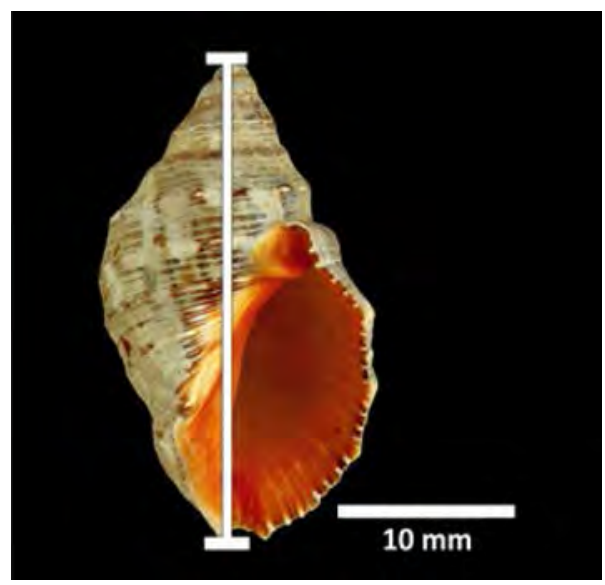

Figura 2. Medida de la altura de Stramonita haemastoma.

\section{RESULTADOS}

\subsection{AnÁlisis de la abundancia de StRamonita HaEMASTOMA}

Se recolectaron un total de 337 individuos, de los cuales 267 se encontraron en la Finca El Apio y los 70 restantes en la Punta del Hidalgo (figura 3). Según el análisis PERANOVA para la variable dependiente 'Abundancia de Stramonita haemastoma'varía en función del factor 'Localidad' (tabla 1) revelando una abundancia mucho mayor en la localidad de la Finca El Apio (figura 3).

TABLA 1. RESULTADOS DEL ANÁLISIS DE LA VARIANZA POR PERMUTACIONES DE TRES VÍAS DE LA 'ABUNDANCIA DE STRAMONITA HAEMASTOMA' EN FUNCIÓN DE TRES FACTORES (LOCALIDAD (LO), DISTANCIA AL IMPACTO (DIS), NIVEL DE MAREA (NI) Y LA INTERACCIÓN ENTRE ELLOS (LOxDIS; LOxNI; DISxNI; LOxDISxNI)

\begin{tabular}{lccccc}
\hline FUENTE DE VARIACIÓN & DF & SS & MS & PSEUDO-F & P (PERM) \\
\hline LO & 1 & 1111,10 & 1111,10 & 9,82 & 0,003 \\
\hline DIS & 2 & 100,72 & 50,36 & 0,44 & 0,666 \\
\hline NI & 1 & 324 & 324 & 2,86 & 0,104 \\
\hline LOxDIS & 2 & 448,39 & 224,19 & 1,98 & 0,156 \\
\hline LOxNI & 1 & 106,78 & 106,78 & 0,94 & 0,354 \\
\hline DISxNI & 2 & 615,17 & 307,58 & 2,71 & 0,082 \\
\hline LOxDISxNI & 2 & 513,72 & 256,86 & 2,27 & 0,135 \\
\hline RES & 24 & 2715,30 & 113,14 & & \\
\hline TOTAL & 35 & 5935,20 & & & \\
\hline
\end{tabular}




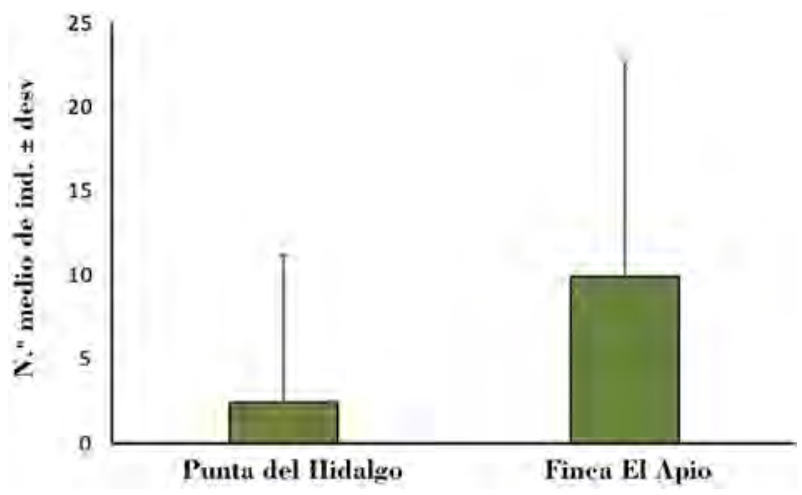

Figura 3. Abundancia de Stramonita haemastoma en las dos localidades estudiadas del Norte de Tenerife.

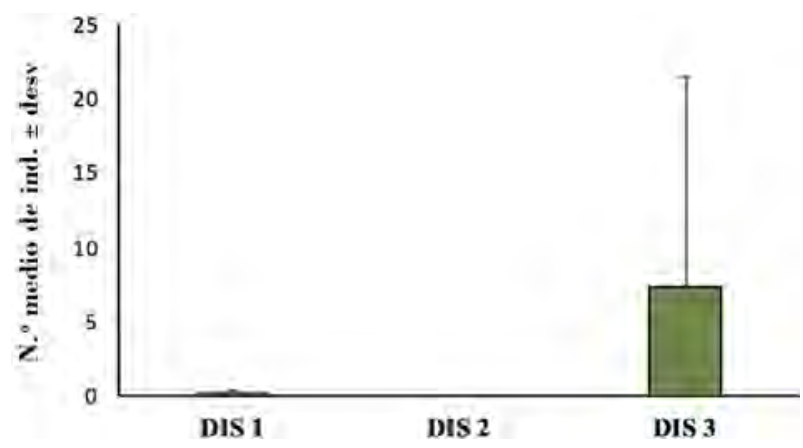

Figura 4. Abundancia de Stramonita haemastoma en las diferentes zonas sometidas a un grado de impacto distinto en función de la distancia al núcleo urbano en la localidad de la Punta del Hidalgo.

Además, los análisis de los datos de las localidades por separado revelan, por un lado, que en la Punta del Hidalgo se observa un aumento significativo de la abundancia (tabla 2) a medida que nos alejamos de dicho impacto (figura 4) y que en la Finca El Apio, por el contrario, encontramos diferencias significativas entre los diferentes niveles de marea (tabla 3), existiendo una mayor abundancia en el mesolitoral que en infralitoral (figura 5).

\subsection{Análisis de las tallas de StRamonita HaEMastoma}

Se realizó un análisis de las tallas de los individuos recolectados en las diferentes localidades, y las frecuencias de los rangos de tallas fueron representados en la figura 6. Tal y como se puede observar, existe una mayor frecuencia de tallas 


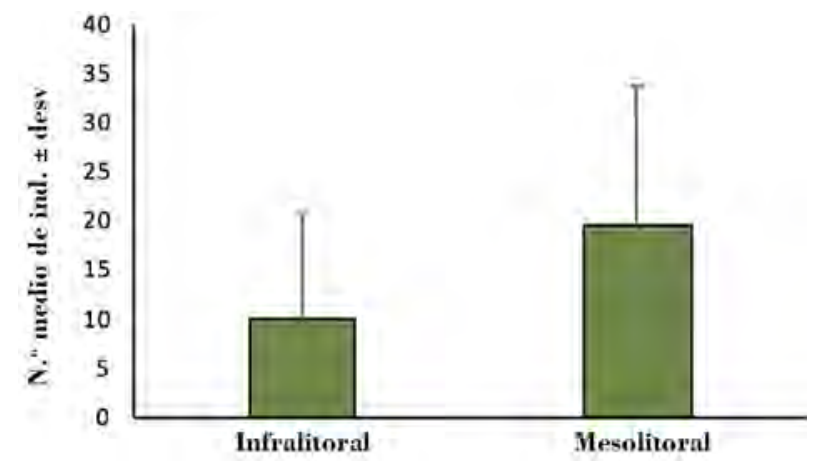

Figura 5. Abundancia de Stramonita haemastoma en los niveles de marea en la localidad de la Finca El Apio.

\begin{tabular}{|c|c|c|c|c|c|}
\hline FUENTE DE VARIACIÓN & DF & SS & MS & PSEUDO-F & P (PERM) \\
\hline DIS & 2 & 16,40 & 8,20 & 5,39 & 0,007 \\
\hline NI & 2 & 5,02 & 2,51 & 1,65 & 0,200 \\
\hline DISxNI & 2 & 5,02 & 2,51 & 1,65 & 0,200 \\
\hline RES & 18 & 27,36 & 1,52 & & \\
\hline TOTAL & 26 & 57,09 & & & \\
\hline
\end{tabular}

TABLA 3. RESULTADOS DEL ANÁLISIS DE LA VARIANZA POR PERMUTACIONES DE DOS VÍAS DE LA 'ABUNDANCIA DE STRAMONITA HAEMASTOMA' EN LA LOCALIDAD DE FINCA EL APIO, EN FUNCIÓN DE DOS FACTORES: DISTANCIA AL IMPACTO (DIS), NIVEL DE MAREA (NI) Y LA INTERACCIÓN ENTRE ELLOS (DISxNI)

\begin{tabular}{lccccc}
\hline FuENTE DE VARIACIÓN & DF & SS & MS & PSEUDO-F & P (PERM) \\
\hline DIS & 2 & 1,10 & 0,55 & 0,42 & 0,691 \\
\hline NI & 2 & 79,89 & 39,94 & 30,67 & 0,001 \\
\hline DISXNI & 4 & 18,12 & 4,53 & 3,47 & 0,051 \\
\hline RES & 18 & 23,44 & 1,30 & & \\
\hline TOTAL & 26 & 122,56 & & & \\
\hline
\end{tabular}

entre los 20-25 mm de altura en la Punta del Hidalgo, mientras que en la Finca El Apio predomina el rango $25-30 \mathrm{~mm}$. No obstante, comprobamos si estas diferencias observables son significativas con la prueba de la $\chi^{2}$ de Pearson (nivel de significación 


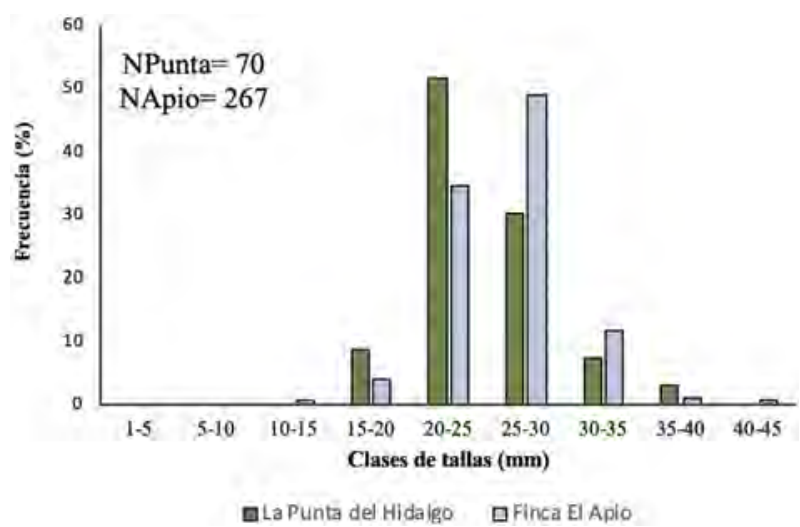

Figura 6. Frecuencia de tallas $(\mathrm{mm})$ de Stramonita haemastoma en las dos zonas de estudio. NPunta: número de individuos recolectados en la Punta del Hidalgo y NApio: número de individuos recolectados en la Finca El Apio.

$0,01)$. El valor resultante, $p=0,021>\alpha=0,01$ no es significativo, por lo que aceptamos la hipótesis nula de independencia.

\section{DISCUSIÓN}

El presente estudio ha revelado la existencia de diferencias en la abundancia de Stramonita haemastoma entre las poblaciones de la Punta del Hidalgo y la Finca El Apio. La Punta del Hidalgo es un lugar cuyo acceso al público no supone dificultad, factor que habitualmente acarrea una mayor presión antrópica en los ecosistemas (Ramírez et al. 2009). De hecho, su enorme heterogeneidad y la cercanía al pueblo favorecen actividades como la pesca y el marisqueo. La Finca El Apio, en cambio, es un lugar de difícil acceso donde no se dan altas concentraciones humanas y que por lo tanto está sometido a un menor impacto antropogénico. Expuestas las particularidades de cada localidad, no resulta sorprendente que esta especie, tan explotada en las islas Canarias, presente una mayor abundancia en la Finca El Apio respecto a la Punta del Hidalgo.

Al estudiar cada localidad de forma independiente, comprobamos que en la Punta del Hidalgo se observan diferencias en la abundancia en relación con la distancia al impacto, verificándose que en la zona más cercana al núcleo urbano y por consiguiente con mayor impacto no se encuentra presente. Por el contrario, en la Finca El Apio no se aprecian cambios respecto a la distancia al impacto ya que la abundancia se mantiene constante en todas las zonas. Sin embargo, respecto al nivel de marea podemos observar una mayor abundancia en la franja mesolitoral al compararlo con la infralitoral. Este último dato se corrobora con las observaciones de otros autores, quienes señalaron que el patrón de distribución general de este 
molusco se extiende por el mesolitoral (Ramírez et al. 2009; Santos y Boehs 2010). A pesar de esto, en la Punta del Hidalgo, Stramonita haemastoma está ausente en el mesolitoral de las zonas más cercanas al núcleo urbano, apareciendo únicamente en el infralitoral.

De este modo, concluimos que el impacto humano puede estar alterando la distribución de la especie debido a que los individuos presentes en el mesolitoral suponen un objetivo de captura más factible. Teniendo en cuenta la veda establecida por el Gobierno de Canarias, la disminución de la cańadilla en el intermareal podría deberse a la búsqueda de alimento cuando la presión marisquera no opera directamente sobre esta especie sino sobre su alimento, por ejemplo, las lapas (Rilov et al. 2002).

Con respecto al tamaño de los individuos hemos podido observar que predominan distintos rangos de talla en cada localidad, siendo la Finca El Apio el lugar donde encontramos ejemplares de mayor tamaño. Según el estudio de Ramírez et al. (2009) el rango de talla más frecuente en las islas Canarias es $21-25 \mathrm{~mm}$, lo cual concuerda con los datos recogidos en la Punta del Hidalgo. Sin embargo, en la Finca El Apio existe una mayor frecuencia de individuos entre las tallas $25-30 \mathrm{~mm}$. Esta diferencia puede ser entendida como una alteración de las tallas presentes en los lugares afectados debida a la explotación de individuos de mayor tamaño. Según Roy et al. (2003), el marisqueo es una de las principales causas que provocan la disminución de la talla de los moluscos puesto que los individuos más grandes son preferentemente recolectados, pudiendo ser la Punta del Hidalgo un claro ejemplo de ello.

A modo de conclusión, en este estudio se ha puesto de manifiesto que el impacto antrópico ocasiona una disminución en la abundancia y el tamaño de los individuos de Stramonita haemastoma, al igual que cambios en la distribución a lo largo del intermareal, lo cual puede ser debido a la tradición de marisqueo en la Isla. En este contexto, teniendo en cuenta las interacciones ecológicas de Stramonita haemastoma con otros organismos, que también se ven afectados por el marisqueo, en especial sus potenciales presas, este estudio podría ser complementado en un futuro mediante un estudio comparativo a largo plazo del impacto antrópico sobre dichas especies. Por último, nos gustaría señalar que estos resultados pueden ser valiosos para una gestión adecuada y sostenible de este recurso.

\section{AGRADECIMIENTOS}

Queremos agradecer al doctor José Carlos Hernández la ayuda y tutorización prestada a la hora de realizar este trabajo, también a aquellos compañeros del Máster de Biología Marina: Biodiversidad y Conservación que nos brindaron su ayuda durante los muestreos y al Departamento de Biología Animal, Edafología y Geología de la ULL por los materiales aportados. 


\section{CONTRIBUCIÓN DE LOS AUTORES}

Conceptualización: EC.

Metodología y trabajo de campo: EC, PC, GML, RM, GDS.

Análisis de datos: GDS, PC, GML, EC, RM con ayuda del profesor José Carlos Hernández.

Preparación del escrito original: EC, PC, GML, RM, GDS.

Corrección y edición del escrito definitivo: GDS.

\section{REFERENCIAS}

Anderson, M.J. 2001. Permutation tests for univariate or multivariate analysis of variance and regression. Can. J. Fish. Aquat. Sci. 58: 626-639.

Elejabeitia, Y., Afonso-Carrillo, J. 1994. Observaciones sobre la zonificación de las algas de Punta Hidalgo, Tenerife (Islas Canarias). Anu. Estud. Canarios. 38: 15-23.

Hernández, E.M.M. 2006. Las arqueomalacofaunas en contextos prehistóricos de Tenerife. Coloq. Hist. C. Am. 17(17): 414-452.

Orden n. ${ }^{\circ}$ 2492. BOC n. ${ }^{\circ}$ 93. Consejería de Agricultura, Ganadería, Pesca y Medio Ambiente. Canarias, 11 de mayo de 2011.

Ramírez, R., Tuya, F. y Haroun, R.J. 2008. El Intermareal Canario. Poblaciones de lapas, burgados y cańadillas. BIOGES, Universidad de Las Palmas de Gran Canaria, p. 54.

Ramírez, R., Tuya, F. y Haroun, R.J. 2009. Spatial patterns in the population structure of the whelk Stramonita haemastoma (Linnaeus, 1766) (Gastropoda: Muricidae) in the Canarian Archipelago (eastern Atlantic). Sci. Mar. 73(3): 431-437.

Roy, K., Collins, A.G., Beckerecker, B.J., Begovic, E. y Engle, J.M. 2003. Anthropogenic impacts and historical decline in body size of rocky intertidal gastropods in southern California. Ecol. Lett. 6(3): 205-211.

Rilov, G., Benayahu, Y. y Gasith, A. 2001. Low abundance and skewed population structure of the whelk Stramonita haemastoma along the Israeli Mediterranean coast. Mar. Ecol. Prog. Ser. 218: 189-202.

Santos, J.J.B. y Boens, G. 2011. Spatial-temporal distribution and recruitment of Stramonita haemastoma (Linnaeus, 1758) (Mollusca) on a sandstone bank in Ilhéus, Bahia, Brazil. Braz. J. Biol. 71(4): 799-805.

Stephenson, T.A. y Stephenson, A. 1949. The universal features of zonation between tide-marks on rocky coasts. J. Ecol. 37: 289-305. 